\title{
Identidad Docente frente a la Calidad como Estandarización en las Escuelas de la Región del Biobío
}

\author{
Teaching Identity for Quality as Standardization in the Schools \\ of the Biobio Region
}

\author{
Donatila Ferrada * \\ Universidad Católica del Maule
}

\begin{abstract}
El fenómeno de homologación de la calidad de la educación con estandarización y homogenización de la población escolar producido por el sistema educativo chileno adscrito a un modelo de mercado, ha modificado las características identitarias de los docentes como respuesta de adaptación y resistencia de un ejercicio laboral que demanda la consideración situacional de territorio, cultura y lengua. Este artículo, recoge resultados de una investigación de carácter participativo, que problematiza sobre esta cuestión y desde las propias voces del profesorado reconstruye esas características identitarias, constituyendo con ellos una comunidad de investigación compuesta por 30 docentes de la región del Biobío que asumió la totalidad del proceso investigativo. Los hallazgos permitieron construir las tipologías de identidad docente en contexto indígena, identidad docente como resistencia a la imposición del Estado, identidad docente frente a los riesgos que enfrenta la profesión, e identidad docente desde el rol formador de ciudadanos situados.
\end{abstract}

Descriptores: Calidad de la educación, Control de rendimiento escolar, Identidad cultural.

The phenomenon of homologation of the quality of education with the standardization and homogenization of the school population produced by the Chilean educational system assigned to a business model has modified the identity characteristics of teachers as a response of adaptation and resistance of work practice that demands the situational consideration of territory, culture and language. This article shows the results of a participatory research that poses this problematic theme and from the teachers' own voices reconstructs these identity characteristics, making up with them a research community composed by 30 teachers from the Biobío region who took over the whole research process. The findings made it possible to construct the typologies of teacher identity in an indigenous context, teaching identity as resistance to the imposition of the State, teaching identity against the risks faced by the profession, and teacher identity from the role of educating situated citizens.

Keywords: Educational quality, Educational testing, Cultural identity.

Proyectos Fondecyt 1140363 "Competencias para la formación de profesores para un desempeño de calidad en vulnerabilidad escolar desde sus propios protagonistas"

*Contacto: dferrada@ucm.cl

ISSN: $0718-7378$

www.rinace.net/rlei/
Recibido: $\quad 18 / 12 / 2016$

$1^{\text {a }}$ Evaluación: 27/01/2017

Aceptado: 28/03/2017 


\section{Antecedentes de la problemática}

Calidad, según el diccionario de la real academia española, proviene del latín qualitas, y es una propiedad o conjunto de propiedades inherentes a algo, que permiten juzgar su valor (buena o mala) y según el diccionario de sinónimos y antónimos, son sinónimos de ella clase, carácter, categoría, jerarquía, característica. De allí que este concepto lleva consigo tanto la valoración de cosas o sistemas, en base a indicadores estratificados que permiten elaborar resultados, y con ellos comparaciones de lo que se trate. De esta forma, el concepto de calidad resulta muy apropiado de usar en disciplinas como la administración y la economía, pues permite generar indicadores para determinar las valoraciones que se persigan conocer y las consecuentes comparaciones entre ellas. Precisamente desde el campo de la economía se comprende la calidad como "la adecuación constante y eficiente de nuestro producto/servicio a las expectativas que del mismo tienen los clientes, de manera que su percepción suponga una ventaja competitiva permanente de nuestra empresa respecto a las alternativas que ofrecen nuestros competidores" (Círculo de Economía, 2012, p. 4). Desde esta comprensión, resulta consecuente elaborar formas de medición estandarizadas que reporten información sobre las cosas (recursos) o sistemas (formas de organización) para determinar, por ejemplo la eficacia o la eficiencia de los mismos, en función de determinar su capacidad competitiva frente a otra empresa.

Desde este simple análisis del concepto de calidad no es posible observar mayor complejidad cuando este se le sitúa al interior del campo de la economía o la administración, la problemática se genera cuando se traslada este concepto de calidad al campo de la educación -campo, que por cierto también cuenta con recursos y sistemas que pueden ser medidos desde estos parámetros de calidad- sin embargo, resulta del todo equívoco cuando se le aplica a aquel espacio de interacción dado en los procesos de enseñanza y aprendizaje entre los sujetos para construir significados y sentidos en torno las materias escolares.

En otras palabras, y de acuerdo con Habermas (1999), en lo que refiere a su teoría de la acción comunicativa sobre el funcionamiento simultáneo de la sociedad tanto como sistema y como mundo de la vida, es posible afirmar que se puede hablar de calidad ${ }^{1}$ cuando referimos al sistema, no así cuando lo hacemos respecto del mundo de la vida, pues el primero tiene como recursos el dinero y el poder, por lo mismo pueden ser traducidos a indicadores, parámetros, categorías, jerarquías, estandarizaciones, etc. En cambio cuando referimos al mundo de la vida, espacio donde se desarrolla la cultura, la sociedad y la personalidad, cuestión que coincide con las funciones encomendadas a la escuela, cuyo recurso es la solidaridad, definitivamente, el concepto de calidad, no aplica.

Desde esta comprensión, entonces, cuando hablamos de calidad de la educación en Chile, en función de los resultados de pruebas estandarizadas que permiten comparar entre los sujetos y sus comunidades, de lo que hablamos es de un poderoso proceso de colonización sobre el mundo de la vida (en la escuela) producido desde el sistema económico neoliberal, así, las categorías del sistema jerarquizan desde sus propios

\footnotetext{
${ }^{1}$ Este estudio reconoce el amplio debate existente en la literatura científica en torno a la calidad de la educación y su amplia difusión, por lo mismo no se adentra en una nueva revisión del mismo, sino que se circunscribe desde la contribución habermasiana de la Teoría de la Acción Comunicativa, en la pretensión de aportar desde una óptica poco explorada.
} 
recursos, las del mundo de la vida, por lo cual en vez de evaluar el sistema, se termina evaluando a los sujetos y sus comunidades, generando con ello, poderosas formas de segregarlos por medio de rótulos cargados de discriminación. Un claro ejemplo de esto es la calidad de la educación a la base de indicadores, donde se asume que todos los sujetos son homogéneos desde sus orígenes, por lo mismo, sus resultados son comparables. Esto lleva consigo, la desconsideración de la heterogeneidad presente en sus comunidades, territorios, cultura y lengua de cada uno de ellos, y no existe corrección estadística posible que pueda cambiar esa condición, toda vez que la estadística se hace cargo de valores objetivables y no del conjunto de la complejidad que implica pertenecer a una comunidad humana u otra. Así, desde los indicadores que conducen a resultados objetivos, se asume una supuesta neutralidad no existente, pues la definición de categorías, criterios, jerarquías que se unifican, siempre obedece a una forma de ver y comprender el mundo, la cual no integra la subjetividad y la complejidad de los sujetos y sus comunidades situadas en cuanto a lo territorial, cultural y lingüístico.

Así, a diferencia de lo que ocurre en el sistema educativo, en el sistema económico o administrativo no opera este proceso colonizador cuando se evalúa su calidad, puesto que sus resultados se mantienen en el espectro de los recursos o la organización de los sistemas, ya sean financieros o administrativos, y muy rara vez son traspasados a los individuos. Un ejemplo anecdótico de esto, es que cuando vamos a realizar un trámite en el banco y no pueden atendernos en línea, el problema es que se cayó el sistema, y no que los sujetos que lo operan son de mala calidad o ineficaces. Por el contrario, cuando los estudiantes que asisten a una escuela obtienen malos resultados a la luz de las pruebas estandarizadoras, el problema son los sujetos (el propio estudiante, el profesor, sus familias, sus comunidades) y no el instrumento de evaluación o las condiciones de su aplicación, etc.

De esta forma, un nuevo lenguaje se instaura en los discursos y prácticas del sistema educativo chileno resultado de esta colonización proveniente del campo de la economía neoliberal, así, conceptos como calidad, estandarización, eficiencia, eficacia, rendición de cuentas, competencias, indicadores, productividad, entre muchos otros, pareciera por algunos momentos, que forman parte incuestionable del cotidiano vivir de las escuelas y sus docentes. En cambio en otros, se constituyen en una temática de alta contingencia, y la escuela en un espacio propicio donde los docentes luchan por descolonizarse de tales conceptos, o donde confrontarse entre ellos respecto de sus diversas posturas. Pero tales conceptos trajeron consigo un sinnúmero de nuevas prácticas que se sumaron como parte de los quehaceres de sus funciones y atribuciones profesionales.

A esta sobrecarga de las tareas docentes en las escuelas, el colectivo docente les ha denominado agobio laboral, definido "como la consecuencia de un conjunto de condiciones que hoy afectan negativamente la labor docente" (Mesa Sobre Ley 20.501, 2016, p. 3) Entre este conjunto de condiciones que definen el agobio laboral ${ }^{2}$, se

${ }^{2}$ Número de alumnos/as por curso, en un porcentaje significativo de escuelas y salas de clase, que no facilita una adecuada atención de la diversidad de estudiantes y ritmos de aprendizaje. Asignación de tareas no lectivas que en muchos casos exceden la jornada laboral contratada, lo que obliga el trabajo fuera de dicha jornada. Realización de un significativo número de tareas administrativas que no están directamente relacionadas con el trabajo pedagógico de los/as docentes. Insuficiente comprensión y respeto de la autonomía profesional docente de una parte importante del sistema escolar, con consecuencias en la imposición de métodos y opciones técnico-pedagógicas que la normativa claramente delimita como espacios de definición de los/as profesores/as (como la planificación). Deterioro del clima y la convivencia escolar en un número significativo de escuelas y liceos, de todas las dependencias y niveles socioeconómicos, con efectos claros en las 
encuentra el efecto de las pruebas estandarizadas en el sistema escolar definido como aquella "presión asociada a la aplicación de un alto número de evaluaciones estandarizadas, con efectos negativos en la comprensión integral de la calidad y en las relaciones y cultura escolar" (Mesa Sobre Ley 20.501, 2016, p. 3) El efecto de los resultados de estas pruebas toca fuertemente a los docentes, produciéndose una "culpabilización de los docentes, en una evaluación social de su trabajo que se efectúa periódicamente a partir de los resultados de aprendizaje obtenidos en mediciones estandarizadas de sus alumnos" (Reyes et al., 2010, p. 271) cuestión que ha contribuido al deterioro de los ambientes de trabajos y de la salud de los docentes.

Marco en el cual se ha generado, entre otros, el movimiento conocido como Alto al SIMCE, expresión de rechazo por parte de los docentes, precisamente, a una de las pruebas estandarizadas más emblemáticas del sistema escolar chileno conocida como Sistema de Medición de la Calidad Educativa (SIMCE) creado en 1988 y vigente hasta nuestros días. Sus defensores plantean que el movimiento Alto al SIMCE "busca visibilizar el problema profundo detrás del SIMCE en sus dimensiones política, humana y técnico-pedagógica. Creemos que solo reconociendo el problema en todas sus dimensiones podemos pensar en alternativas coherentes con las demandas por una educación como un derecho" (Salinas y Insunza, 2013)

Como contraparte, el propósito del SIMCE, de acuerdo a lo declarado por el Ministerio de Educación chileno es "contribuir al mejoramiento de la calidad y equidad de la educación, informando sobre los logros de aprendizaje de los estudiantes en diferentes áreas de aprendizaje del currículo nacional" (Agencia de Calidad de la Educación, 2016, p. 1) Sin embargo, los resultados de esta prueba, han constatado año a año la profunda segregación del sistema escolar chileno en directa relación con la dependencia administrativa de las escuelas ${ }^{3}$, que a su vez agrupan a los diversos sectores socioeconómicos de la población nacional, a saber, escuelas privadas, escuelas subvencionadas y escuelas públicas, siendo las primeras las que obtiene mejores logros y las públicas los peores. De esta forma, con los resultados de esta prueba, se elaboran ranking que estratifican las escuelas, los docentes, los estudiantes y sus comunidades.

Desde este contexto, cabe preguntarse si estos procesos de colonización marcados por el concepto de calidad asociado a la estandarización sobre el espacio escolar están afectando las identidades colectivas de los docentes que se desempeñan en diversidad de contextos culturales, lingüísticos y territoriales.

\section{Marco referencial}

Diversos autores coinciden en plantear que la identidad sólo puede ser individual aunque portadora de una dimensión intersubjetiva y relacional. De acuerdo con Pirrone (2006, p. 2), la identidad se caracteriza por "la distinguibilidad, es decir, la posibilidad o la necesidad de ser particulares...Es la autopercepción de un sujeto en relación con los

condiciones laborales de los equipos docentes. Insuficientes espacios formales de participación de los/as profesores/as en decisiones relevantes de sus establecimientos, lo que resta potencial a la gestión educativa y a la adhesión de los actores a los proyectos educativos. Ejecución de distintos sistemas (nacionales y locales) de evaluación docente, que suponen una fuerte demanda de tiempo para los/as profesores/as y que mayoritariamente exceden la jornada laboral.

s Para una profundización al respecto visitar el portal de la Agencia de Calidad del Ministerio de Educación www.agenciaeducacion.cl 
otros; a lo que corresponde, a su vez, el reconocimiento y la aprobación de los otros sujetos". En la misma idea se plantea Toledo (2012, p. 46) cuando afirma que "la identidad es un sentido otorgado por el sujeto a su propia experiencia, la identidad no puede ser compartida...la identidad se construye en la relación del sujeto con su entorno y con los otros".

Siguiendo a Pirrone (2006), esta característica primordial de la identidad se sustentaría en tres pilares básicos, a saber, la pertenencia social, los atributos identificadores y la narrativa biográfica del sujeto. Del primer pilar, el sujeto obtiene la pluralidad de sus pertenencias sociales a grupos, redes, categorías, etc., con quienes comparte -al menos en parte- el núcleo de representaciones sociales de esa comunidad, lo que le otorga un marco para percibir e interpretar la realidad, al mismo tiempo que formas de comportarse e interactuar con los demás miembros del colectivo social. Del segundo pilar, el sujeto obtiene la configuración de los atributos que lo distinguen, tales como, sus hábitos, disposiciones, imagen corpórea, con los cuales se presenta en la interacción social frente a los demás. Y del tercer pilar, el sujeto obtiene la construcción de sentido otorgado a partir de su propia trayectoria de vida, que requiere ser confrontada desde la perspectiva de los otros. A este proceso también se le denomina como identidad íntima o biográfica.

Desde estos antecedentes que definen la identidad personal o la identidad como un atributo del sujeto individual cabe preguntarse, ¿se puede hablar de identidades colectivas? Al respecto, Toledo (2012) advierte que la identidad involucra el sentido dado por el sujeto a su propia experiencia, ella no puede ser compartida. Si bien se construye en la interacción, en un contexto, en un territorio, en una historia, la identidad nunca es colectiva, aunque sea el colectivo lo que le permite al sujeto construir su identidad.

Pirrone (2006) no comparte totalmente lo anterior, pues afirma que podría hablarse de identidades colectivas "si es posible concebir actores colectivos propiamente dichos, sin necesidad de hipostasiarlos ni de considerarlos como entidades independientes de los individuos que los constituyen” (p. 4). Se trataría de entidades relacionales de sujetos con un sentido común de pertenencia y con una orientación común de la acción. De esta forma se trataría de "actores colectivos capaces de pensar, hablar y operar a través de sus miembros o de sus representantes según el conocido mecanismo de la delegación (real o supuesta)" (p. 4).

En esta misma idea Bartolomé (2006) asume la existencia de las identidades colectivas cuando estudia las identidades étnicas, entendiéndola como una construcción "resultante de una estructuración ideológica de las representaciones colectivas derivadas de la relación diádica y contrastiva entre un nosotros y un los otros" (p. 29). De esta forma hablaríamos de sujeto-grupo o de sujeto-actor colectivo. Sin embargo, Pirrone (2006) recomienda tener presente las siguientes proposiciones axiomáticas sobre las identidades colectivas, a fin de evitar equívocos al respecto:

1) Sus condiciones sociales de posibilidad son las mismas que las que condicionan la formación de todo grupo social: la proximidad de los agentes individuales en el espacio social.

2) La formación de las identidades colectivas no implica en absoluto que éstas se hallen vinculadas a la existencia de un grupo organizado. 
3) Existe una distinción inadecuada entre agentes colectivos e identidades colectivas, en la medida en que éstas sólo constituyen la dimensión subjetiva de los primeros, y no su expresión exhaustiva. Por lo tanto, la identidad colectiva no es sinónimo de actor social.

4) No todos los actores de una acción colectiva comparten unívocamente y en el mismo grado las representaciones sociales que definen subjetivamente la identidad colectiva de su grupo de pertenencia.

5) Frecuentemente las identidades colectivas constituyen uno de los prerrequisitos de la acción colectiva. Pero de aquí no se infiere que toda identidad colectiva genere siempre una acción colectiva, ni que ésta tenga siempre por fuente obligada una identidad colectiva.

6) Las identidades colectivas no tienen necesariamente por efecto la despersonalización y la uniformización de los comportamientos individuales (salvo en el caso de las llamadas instituciones totales, como un monasterio o una institución carcelaria). (Pirrone, 2006, pp. 4-5)

Desde este marco de comprensión de identidades colectivas, la ocupación de este estudio sobre el efecto de los procesos de colonización del lenguaje del mundo de la economía neoliberal sobre el trabajo docente, expresado en este caso desde el concepto de calidad otorgados por las pruebas estandarizadas, requiere iniciar reconociendo que "la identidad profesional del pedagogo, como la de cualquier otro profesional o como la de cualquier otro sujeto es relacional, histórica e híbrida" (Navarrete, 2008, p. 522). Esta autora plantea que la identidad colectiva de los docentes es relacional porque se construye en un marco de fundamentos teóricos que comparte con otras profesiones, como por ejemplo, con la psicología, la sociología, con la filosofía, y eso también se vincula con el campo laboral de desempeño del docente. Así también, la identidad colectiva de los docentes se caracteriza por ser cambiante, ambigua, fracturada y en muchos aspectos temporal, pues depende de las decisiones que ellos tomen en su contexto histórico-social.

Una investigación sobre el ser docente, que se desarrolló con profesores chilenos que están viviendo los efectos de un modelo educativo de mercado que los supervigila y supercontrola en sus espacios laborales, reafirma la idea que la identidad docente se construye desde la situacionalidad histórico social en la cual se encuentran los miembros del colectivo. Este estudio concluye que:

\begin{abstract}
lo colectivo cobra vital importancia, en tanto ayuda a la contención, a la generación del sentimiento de no estar solos y de fortalecerse...Así mismo, contribuye a la comprensión y re construcción del sentido del propio quehacer y de sí en tanto docente...Emergen, así, espacios y ejes de acción que se resignifican como propios, esto es, la lucha politica por la autonomía docente y la autoridad profesional que posibilita el trabajo pedagógico libre y creativo. (Reyes et al., 2010, pp. 285-286)
\end{abstract}

\title{
2. El contexto y la forma de investigar
}

En términos metodológicos, se optó por trabajar por lo que hoy se reconoce como paradigma participativo (Gayá Wicks y Reason, 2009; Guba y Lincoln, 2012; Heron y Reason, 1997) cuyo eje central descansa en una realidad subjetiva-objetiva, cocreada por la mente y el universo dado, de tal forma que se realiza una transacción participativa entre la subjetividad crítica del sujeto y el universo, donde el conocimiento experiencial, proposicional y práctico de cada uno de los integrantes de comunidades de investigación producen hallazgos cocreados. Al interior de este paradigma se optó por la investigación dialógica-kishu kimkelay ta che (Ferrada et al., 2014), cuya traducción literal desde la 
lengua chedungun (propia del pueblo mapuche presente en la región del Biobío) significa: ninguna persona conoce y/o aprende por sí misma. Así, el conocimiento no es un acto creador individual sino, por un lado, deviene de generaciones pasadas, en cuanto criterio temporal, y, por otro, es una creación colectiva y, por tanto patrimonio social y cultural dinámico del colectivo (Durán, Catriquir y Berho, 2011)

Desde esta comprensión, la investigación dialógica-kishu kimkelay ta che, se organiza en torno a la conformación de comunidades de investigación quienes deciden qué, porqué, para qué y cómo investigar, teniendo presente que el carácter cualitativo de este estudio circunscribe los resultados al contexto considerado. Para ello, resultó primordial constituirse como sujetos situados, y constituidos como comunidad de investigación, que a lo largo de los 3 años que duró la investigación logró integrar a 30 profesores de la región del Biobío en Chile, con un promedio de 23 años de ejercicio laboral. Todos estos docentes de enseñanza básica de escuelas públicas chilenas pertenecientes a 8 comunas de la región del Biobío, compartían un criterio de distinción que fue la diversidad de contextos de desempeño laboral y un criterio de unificación que fue el alto índice de vulnerabilidad educativa (IVE) de las escuelas en que se desempeñaban de acuerdo con los datos del Ministerio de Educación chileno (JUNAEB, 2016). La distribución de los docentes en los contextos y escuelas, se muestra la tabla 1.

Tabla 1. Docentes situados en sus contextos laborales

\begin{tabular}{|c|c|c|}
\hline $\mathrm{N}^{\circ}$ DOCENTES & $\begin{array}{c}\text { CONTEXTO DE DESEMPEÑO } \\
\text { LABORAL }\end{array}$ & $\begin{array}{c}\text { CARACTERÍSTICAS DE LAS } \\
\text { ESCUELAS }\end{array}$ \\
\hline $\begin{array}{l}6 \text { docentes } \\
\text { proveniente de } 2 \\
\text { escuelas }\end{array}$ & $\begin{array}{l}\text { Alta población indígena Lafkenche, } \\
\text { rural en la comuna de Alto Biobío. } \\
\text { Alta población indígena } \\
\text { Pehuenche, rural en la comuna de } \\
\text { Tirúa. }\end{array}$ & $\begin{array}{l}\text { Escuelas básicas con programa } \\
\text { intercultural bilingüe, con } \mathrm{IVE}=99.0 \\
\text { y } 98.0\end{array}$ \\
\hline $\begin{array}{l}16 \text { docentes } \\
\text { provenientes de } 4 \\
\text { escuelas }\end{array}$ & $\begin{array}{l}\text { Zonas altamente urbanizadas, sin } \\
\text { población indígena en las comunas } \\
\text { de Los Ángeles, Concepción, } \\
\text { Chillán y Lebu. }\end{array}$ & $\begin{array}{l}\text { Escuelas básicas con programa de } \\
\text { acompañamiento compartido (PAC), } \\
\text { con IVE }=87.0 ; 88,9 ; 91.2 ; 86.3\end{array}$ \\
\hline $\begin{array}{l}8 \text { docentes } \\
\text { provenientes de } 2 \\
\text { escuelas }\end{array}$ & $\begin{array}{l}\text { Zonas rurales sin población } \\
\text { indígena en las comunas de El } \\
\text { Carmen y Trehuaco }\end{array}$ & $\begin{array}{l}\text { Escuelas con programa de } \\
\text { acompañamiento compartido (PAC), } \\
\text { con IVE }=92.5 \text { y } 95.7\end{array}$ \\
\hline
\end{tabular}

Fuente: Elaboración propia.

Estos 30 docentes conformaron la comunidad de investigación que realizó la problematización situada en sus propios contextos de desempeño laboral, quedando circunscrita en torno a la pregunta ¿de qué forma se está viendo afectada la identidad docente por el impacto en sus labores cotidianas del concepto de la calidad educativa estandarizada propuesta desde el Estado?. Para tales efectos, se seleccionaron los procedimientos de producción del conocimiento conversación dialógica y diálogos colectivos, propios de este tipo de investigación. Los procesos de análisis e interpretación se realizaron en conjunto con la propia comunidad de investigación.

\section{Resultados: Características identitarias de los docentes}

El profesorado situado en la región del Biobío, se pronuncia vívidamente desde sus condiciones laborales frente al concepto de calidad de la educación instaurado en Chile, 
en los términos ya descritos de estandarización, de indicadores, categorías, jerarquías, desde los cuales se construyen las categorías de valoración, ya sea como buena, mediana o mala calidad educativa de las escuelas, de los directivos, de los profesores, de los estudiantes y en última instancia de las familias. Sobre todo, en cuanto a la extrapolación de los resultados que se realizan y que cruzan todos los niveles de análisis, no estando presentes la totalidad de ellos en los propios indicadores que se consideran para medir la calidad educativa. Especialmente relevante resulta en sus diálogos, lo que ocurre con el SIMCE, que evalúa el resultado de materias escolares (asignaturas de lenguaje, matemática y ciencias) en estudiantes de diversos niveles, cuyos resultados permiten extrapolarse hacia la calidad de las escuelas, del profesorado, del equipo directivo, personas e instituciones que no han sido consideradas en la medición. De allí entonces, el profesorado participante de la investigación sostiene que toda vez que se implementa un sistema de medición de la calidad de la educación desde el Estado, las preguntas claves a responderse son por qué? para qué? para quién/es? quién/es la definen? con qué intereses? desde dónde se posicionan? por qué estas decisiones no son debatidas? por qué se imponen? Claro está que las respuestas a estas cuestiones no se insertan en la consideración de los sujetos situados en un territorio, en una cultura, en una lengua. Con ello, es posible evidenciar un primer carácter colectivo que los caracteriza y que les otorga una identidad propia del colectivo docente.

Sin embargo lo anterior, la experiencia de trabajo con la comunidad de investigación, demostró que el colectivo docente resultó ser mucho más dinámico de lo que sus propios miembros pensaban, quienes inicialmente se autorrepresentaban como un colectivo más bien homogéneo. Cuestión que durante el transcurrir del trabajo se fue manifestando una diversidad de agrupaciones donde sus miembros se movilizaban al interior de unas, en algunas ocasiones, en cambio en otras no se sentían representados. Desde el reconocimiento de esta permanente dinámica se acordó la identificación de cuatro características identitarias diferenciadoras al interior del colectivo docente que componía la comunidad de investigación, al interior de los cuales se ubicaban claramente docentes que mayoritariamente compartían características, y algunos otros que no, a saber: 1) identidad docente en contexto indígena, 2) identidad docente como resistencia a la imposición del Estado, 3) identidad docente frente a los riesgos que enfrenta la profesión, e 4) identidad docente desde el rol formador de ciudadanos situados.

\subsection{Identidad docente en contexto indígena}

La impronta de la medición de la calidad de la educación resulta fuertemente cuestionada desde el conocimiento vivo del profesorado situado desde el territorio, la cultura y la lengua mapuche, constituyéndose y distinguiéndose en un colectivo de docentes con una identidad común (algunos de ellos miembros del pueblo mapuche y otros chilenos). El siguiente fragmento de diálogo, muestra esta situación:

...aquí vienen y aplican las pruebas como el SIMCE, pruebas en castellano, con preguntas de la cultura chilena y de otras partes del mundo, las aplican con unos tiempos definidos. Ellos no saben, por ejemplo, que los niños pehuenches son más lentos para todo, porque la vida aquí es más lenta, los animales deben caminar lento porque viven en altura, aquí en la cordillera, y por eso no se le puede apurar, entonces, los niños no es que no sepan contestar esas pruebas, sino que no alcanzan, porque ellos no tienen apuro, su cultura no opera por el tiempo lineal, es circular. Luego de eso, salen los resultados y dicen que nuestra escuela es de mala calidad, que somos malos profesores, un año nos pusimos de acuerdo con los niños y les pedimos que respondieran el SIMCE en chedungun, y a la escuela la sancionaron, sacaron 
hasta directora por eso... esto no es calidad es desconocimiento e imposición, pero sin embargo, nosotros seguimos unidos como profesores de estas escuelas. (Fragmento de dialogo colectivo, Alto Biobío)

Este relato pone en evidencia, los efectos directos sobre los docentes, que se posicionan desde el contexto en que se desempeñan, relevando la especificidad del trabajo en una escuela en que sus estudiantes (y algunos docentes) pertenecen a otra cultura y otra lengua, cuestión que el concepto de calidad estandarizadora sencillamente omite como realidad existente en el contexto nacional, cuyos resultados que además de estigmatizar y rotular, entregan información errónea sobre el real rendimiento académico de estos escolares, pues al no considerar el factor tiempo, se imposibilita acceder al dato certero, de esta forma, esta escuela, sus directivos, su profesorado, su alumnado y su cultura y lengua queda clasificada como de mala calidad.

El desencuentro entre la calidad educativa como estandarización impuesta como realidad única chilena, en cuanto a la lengua castellana y la cultura occidental, con la calidad educativa contextualizada que demanda la consideración, el conocimiento y la legitimación de la lengua y la cultura mapuche, puede verse en el siguiente fragmento:

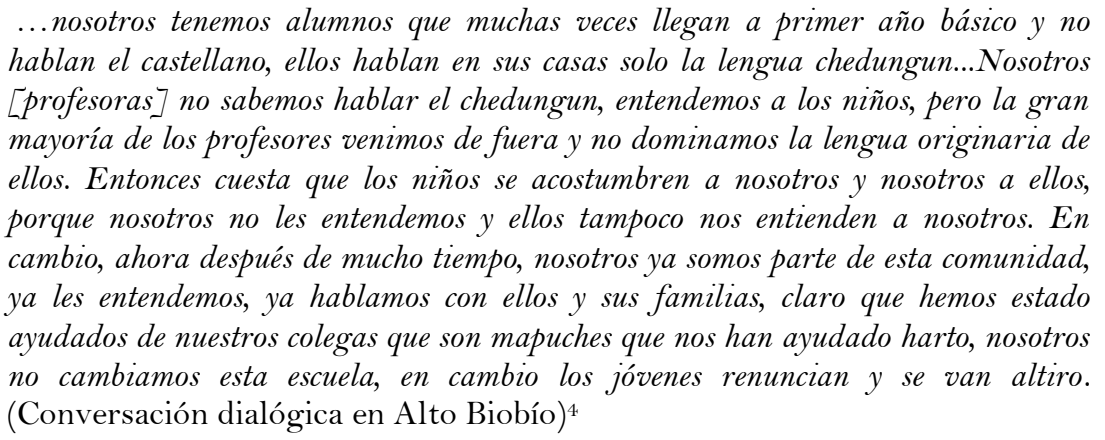

Este fragmento denota el carácter inconcluso que tienen los procesos identitarios, toda vez que aquellos docentes que inicialmente no formaban parte de la comunidad de la escuela pasaron por un proceso en el cual sus autopercepciones y representaciones los ubicaban fuera del lugar, es decir, no formando parte del otro, en cambio, hoy se reconocen como miembro del colectivo de docentes que trabaja y quiere seguir trabajando en ese contexto. Que al mismo tiempo son conscientes de los efectos de una imposición estandarizadora de programas educativos provenientes del estado que deslegitima sus propios espacios laborales, pero que ello se transforma en el eje identitario común a todos los docentes.

$P_{26}: . .$. entonces, vienen del Mineduc y nos imponen el programa PAC [Programa de Apoyo Compartido $]$ con esa estructura rígida de hacer las clases y de considerar los tiempos, pero la realidad de estas escuelas no es la misma que en Santiago, entonces se parte pensando que Chile es igual en todas partes, y por eso se aplican estos programas que los traen desde fuera, sin considerar la realidad nuestra y menos la de nuestros niños pehuenches.

$P_{s . . .}$ Nosotros vemos que están muy equivocados, porque no consideran las características de aquí, entonces nosotros estamos aburridos de eso. Claro que eso en Santiago, en Concepción, en las ciudades grandes, seguramente ayudará mucho a otros docentes, y se ajustará a sus realidades, pero no a la nuestra. (Dialogo Colectivo Alto Biobío-Tirúa)

${ }^{4}$ Parte de este fragmento y parte de los siguientes han sido citados en Ferrada y cols. (2015) Transformar la formación. Las voces del profesorado. Santiago, RIL Editores. 
El proceso identitario aquí se fortalece en torno a la idea de pertenencia al lugar de trabajo que tiene características únicas que deben ser reconocidas, y que deben ser visibilizadas. Características que van configurando un colectivo docente que comparte experiencias comunes y que se distingue de otros colectivos docentes distintos a ellos, porque laboran en otros espacios que tienen otras connotaciones, pero no por ello deben ser impuestas a todos. Así, el concepto de calidad educativa a la base de la estandarización y homogenización parafraseando a Zemelman (2005), correspondería a una realidad inventada, única, monolingüe, unicultural, que sólo existe en la mente de los diseñadores ministeriales, pero está muy lejos de representar la gran diversidad de territorios, culturas y lenguas presentes en el país. Sin embargo, ello produce y afianza la pertenencia de estos docentes a sus espacios laborales, en este caso el contexto indígena.

\subsection{Identidad docente como resistencia a la imposición del Estado}

La única característica identitaria que agrupó a todos los miembros de la comunidad de investigación, más allá de los contextos específicos de desempeño fue la resistencia que manifiestan los docentes frente a la imposición ministerial de programas de mejoramiento educativo desde la comprensión de que es la realidad la que debe ajustarse al modelo, y no a la inversa.

$P_{\imath . . .}$ nos habían venido a meter un trabajo de chinos, nosotros dijimos, no vamos a
aceptar, nos iban a tener tres semanas de las vacaciones de verano, en algo que era
un zapato chino que íbamos a tener... bueno lo hacemos pero a mitad de año van a
estar todos los profesores con licencia médica, porque van a estar todos estresados,
porque si ya con las clases día a día nos acostamos de madrugada. Porque no es una
sola asignatura la que planificamos diariamente, son tres o cuatro asignaturas que
tenemos en el día, más los talleres, y tenemos que tener las clases día a día de todo, y
ahora nos van a meter este trabajo, o sea amanecimos sentados trabajando y ¿̇cuándo
vamos a dormir?, ¿̇a mitad de año?, estamos reventados...

$P_{1:}$ Sí, la rechazamos, querían que hiciéramos una evaluación de los objetivos con la nueva reforma curricular, había que vincular cada objetivo con las evaluaciones, pero ya ese objetivo, a veces trae hasta veinticinco indicadores y si quería saber si este objetivo fue logrado, yo tengo que evaluar los veinticinco indicadores, para saber si el objetivo lo logré o no lo logré, tengo que hacer veinticinco pruebas para ese objetivo y el otro trae veinticinco más, ya son cincuenta pruebas...

$P_{29:}$ Esto lo traen copiado de otras realidades, otros países, donde los profesores tienen mucho tiempo para preparar clases, mucho tiempo para evaluar, tiene muchos menos niños en sus aulas...esto porque los que hacen esto en Chile no son profesores, son economistas, sociólogos, psicólogos, porque ellos no saben lo que es trabajar aquí en estas condiciones, creen que Chile es igual a lo que ellos han visto cuando han viajado a buscar ideas, entonces desde fuera se todo muy fácil, pero aqui es otra cosa.

(Diálogo colectivo conjunto de docentes)

Este diálogo pone en evidencia cómo reaccionan los docentes frente a las propuestas de soluciones emanadas desde el Estado, que son pensadas desde fuera de los territorios, desde fuera de la comprensión de cómo se desarrolla la labor docente, desde fuera de las condiciones reales del desempeño laboral del profesorado, que no tiene tiempo destinado para realizar detalladas programaciones e innumerables evaluaciones de las mismas. Esta lógica unificadora y descontextualizada es férreamente rechazada por el profesorado en ejercicio laboral en la región del Biobío, y viene a representar las desiguales relaciones de poder desde el cual ejerce el profesorado, pero que en esta ocasión son capaces de generar resistencia a dicha imposición. El pleno consenso del profesorado frente a este tipo de resistencia configura una característica de identidad colectiva de resistencia frente a la imposición. 


\title{
3.3. Identidad docente frente a los riesgos que enfrenta la profesión
}

Otro colectivo de docentes que comparte vivencias laborales comunes son aquellos que lo hacen en escuelas categorizadas por el Estado como de alto índice de vulnerabilidad, específicamente las ubicadas en zonas altamente urbanizadas y marginales. Plantean estar expuestos a un doble riesgo en sus propios espacios laborales, uno, respecto de los riesgos de su propia persona, y el otro, respecto de los riesgos al que están expuestos sus propios estudiantes, quienes dependen del resguardo que de ellos realiza el docente. Los siguientes relatos de los docentes expresan esta situación en varias comunas:

\begin{abstract}
$P_{17 . . .}$ acá, el año pasado hubo un asesinato y el que asesinó, y el hijo del asesinado eran compañeros de curso, en un octavo año, o sea muchas cosas, fuertes cosas, imagínate lo que era esa clase...yo tenía miedo de lo que podía pasar, las familias se enfrentaban aquí mismo fuera de la escuela, entonces trabajar aquí no es lo mismo que hacerlo en una escuela privada... porque claro es muy fácil decir que esta escuela no tiene calidad, que hay que mejorar la calidad.
\end{abstract}

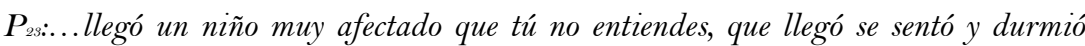
todo el primer bloque... voy a verlo y le pregunto ¿̇qué te pasa?, interrumpo mi clase, ¿qué ocurre? y me dice tía no dormí porque no pude dormir y lo miras bien y viene con un ojo todo negro ¿̇qué hago? continuo mi clase normal? sigo con mi lectura? o me detengo y veo la situación? Obviamente debo interrumpir...entonces no puedo continuar con mis materias y como consecuencia no puedo asegurar la calidad que me piden en el SIMCE, entonces aquí estamos todos muy agobiados con todo esto.

$P_{11}$... yo te digo tengo tres demandas en tribunales con alumnos pequeñitos acá por abuso... Otro ya lleva 60 días o 70 días, sin venir a clases ¿qué hacemos? Tribunales. Entonces tenemos que hacer tanta cosa, ayer no más, vino una pareja de carabineros con el trabajador social y me dice J: ¿̨qué hacemos con F., demandamos?, pero si ya demandamos por abuso... no hagamos más demandas, estoy agotada, porque la madre de ese niño no la hemos podido hacer entender que el niño debe venir a clases, ya que está más protegido acá, además el niño está repitiendo por inasistencia y no porque un niño no sea capaz...tu me dirás como salimos en los resultados cuando miden la calidad en estas escuelas.

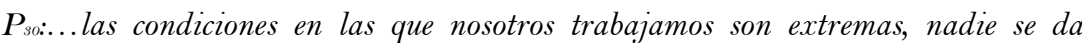
cuenta de esto, nosotros, los de las escuelas de aquí del sector siempre nos juntamos, incluso fuera de las escuelas para conversar, para ayudarnos, porque nos sentimos abandonados. Nosotros sabemos que esta realidad no la tienen todos los colegas, pero no por eso no existe. El otro día tuvimos que ayudar a un colega que fue agredido por un apoderado, entonces creo que estamos traspasando los límites. En estas condiciones laborales no se nos puede evaluar con el SIMCE, con el PAC, con planificaciones diarias, porque donde nosotros estamos, la cotidianidad de nuestro trabajo no lo permite. (Diálogo colectivo entre docentes de Concepción, Chillán, Los Ángeles y Lebu)

Estos relatos reflejan que cuando se piensa la calidad de la educación desde el Estado, es pensada desde la comprensión de una escuela que cuenta con una cohesión social ficticia, desde un concepto de infancia ideal (feliz), desde unas condiciones materiales, emocionales y afectivas dadas (garantizadas), desde un escenario de desempeño laboral libre de conflictos. En otras palabras, desde un concepto teórico de escuela, desde una realidad que sólo existe en la imaginación de quienes no conocen la diversidad presente en muchos lugares del país, no solo de la región del Biobío.

Todas estas vivencias compartidas entre los docentes permiten identificar un colectivo o una identidad colectiva que se reconoce desde la dificultad, desde el riesgo, desde el desamparo y la desprotección en este caso del Estado, por lo mismo, manifiestan rechazo a los procesos estandarizadores de la calidad con los cuales son medidos, toda vez que su 
preocupación central es el cuidado y protección de sus estudiantes y de su propia persona.

\title{
3.4. Identidad docente desde el rol formador de ciudadanos situados
}

Otro colectivo de docentes que se configuró fue aquel proveniente mayoritariamente de sectores rurales y semiurbanos que coincidió de forma especial sobre la necesidad de resituar el rol formador de ciudadanos que ellos tienen, cuestión que en el marco de la estandarización de la educación ha sido trastocado profundamente.

\begin{abstract}
$P_{18:}$ Nosotros estamos formando niños para que se inserten en el mundo global, en el trabajo en igualdad de condiciones, por eso los niños tienen que saber por igual los contenidos de las asignaturas, pero además deben conocer sus propias costumbres, su lengua propia de ellos, su tierra...entonces, estamos hablando de una igualdad educativa que puede ofrecer la escuela para que el niño se inserte en la sociedad global si lo así lo desea, pero que él vaya con su identidad dónde vaya a desempeñarse, o si desea quedarse aqui en su territorio, también sepa que pasa más allá de su propio contexto. Esto no lo consideran estas pruebas que aplican continuamente, estas pruebas solo desvirtúan lo que los niños están aprendiendo. Como vamos a formar buenos ciudadanos con estas pruebas, los niños se forman en la convivencia democrática, en la vivencia diaria del respeto, de la igualdad, todas esas cosas..

$P_{21}$ : Por eso, las pruebas PISA, SIMCE, como que obliga sólo a centrarse en un solo saber, esas pruebas no consideran no valoran la riqueza cultural que hay aquí...tiene que ser así, la escuela no es solo para preparar niños para rendir pruebas, como lo que está pasando en Chile, sino un espacio para que todos los niños aprendan, de sus propia cultura, de la cultura del currículo, de las asignaturas, todo eso a la vez, y eso yo lo considero igualdad educativa...Por eso que, el tema no es de calidad educativa del SIMCE, o de esas pruebas PISA o TIMS, para nosotros eso no es calidad, eso es calidad para ellos, para quienes toman esas decisiones de aplicar tantas pruebas, si ya sabemos las desigualdades que hay en el país, pero ellas no van a resolverse con más pruebas, sino trabajando de otra forma, considerando lo que nosotros también podemos aportar, que tenemos que dar espacio para vivir la democracia en las escuelas, de ejercer la ciudadanía. (Diálogo colectivo, TirúaAlto Biobío)
\end{abstract}

El descontento de los docentes, frente a la gran cantidad de pruebas aplicadas para medir los resultados académicos de los estudiantes, evidencia que lo que se construye desde el Estado como concepto de calidad educativa se agota en estas pruebas estandarizadas, cuyos resultados unifican el sistema educativo, y con ello a los sujetos que forman parte de él. Pero es precisamente esta consecuencia, la que moviliza a los docentes a levantar el concepto de su rol formador capaz de enseñar y poner en prácticas experiencias educativas democráticas, donde sus estudiantes vivencien el significado del ejercicio de ciudadanía como la forma de recuperar todo aquello obviado y omitido en dicha estandarización. Estos conceptos recuperan el valor de su trabajo, de sus escolares, de su escuela, de su comunidad, del territorio y de la cultura de los que también es parte y que comparte. Así, reconceptualizan el rol formador sobre la base de un concepto de igualdad educativa que legitima a los sujetos y a sus contextos, al mismo tiempo que al propio currículum escolar propuesto por el Estado, pero este currículum debe ser resituado, considerar las culturas, las lenguas y los territorios locales, es decir, de quienes son los agentes que le dan vida, y en ese interactuar con él se transforma y se amplía.

\section{Discusión y conclusiones}


Dado el carácter participativo y colectivo de la investigación dialógica-kishu kimkelay ta che, cuestión que adquirió vida en los numerosos encuentros dialógicos producidos entre el profesorado participante de este estudio, y su pretensión de construir conocimiento propositivo en vías de avanzar hacia una transformación de la realidad que se investiga, fue posible alcanzar acuerdos al respecto, que se sintetizan a continuación:

- La comprensión de la calidad de la educación como estandarización impuesta por el sistema educativo chileno adscrito a un modelo de mercado, ha impactado la construcción de identidad colectiva docente llegando a modificar las características identitarias de éstos, como respuesta de adaptación y resistencia de un ejercicio laboral que clama por la consideración situacional de territorio, cultura y lengua en el que se desempeñan cotidianamente.

- Existe una fuerte convicción por parte del profesorado que quienes toman las decisiones respecto de las políticas educativas tanto en cuanto a evaluación de la calidad de la educación en Chile, como de las propuestas de mejora únicas para todo el país, está en manos de pocas personas que se han configurado como expertos, cuyo aval está dado en criterios muy alejados del conocimiento cabal de la realidad educativa regional que se requiere para avanzar en cambios sustantivos en la escuela pública chilena. Estos iluminados o expertos no son legitimados como agentes válidos por este profesorado, pues sus decisiones están muy fuera de las necesidades reales en las cuales ellos se desempeñan. Proponen pasar de las decisiones y definiciones centradas en algunos iluminados que saben lo que hay que hacer a construcciones colectivas de los sujetos situados desde sus propios territorios, lengua y cultura.

- Existe un fuerte descontento, por parte del profesorado frente a las políticas de calidad educativa y de mejoras para alcanzarlas, toda vez que sus propias propuestas no están contempladas desde el Estado, por el contrario, las propuestas son traídas desde otros contextos y otras realidades que no concuerdan con sus propias necesidades, cuestión que sitúa al profesorado en una condición de asistencializado, que no está dispuesto a asumir, por el contrario, se define como autónomo y capaz de decidir con criterios avalados desde sus propia experticia y su sabiduría experiencial en cada contexto de desempeño. Proponen pasar de comprender a las comunidades de forma asistencializada a comprenderlas como colectivos autónomos y capaces de decidir su propio futuro.

- La fuerte clasificación que realiza los resultados de la medición de la calidad de la educación en las escuelas, el profesorado, el estudiantado y sus familias, permite sostener al profesorado que ello solo trae consecuencias negativas que impiden avanzar en igualdad, por lo que resulta urgente abandonar este tipo de construcción. Proponen romper con los estereotipos, estigmatizaciones y categorización sobre los sujetos y sus comunidades que viven en condiciones de desigualdad.

- Existe conciencia que se ha instaurado una cultura de la fatalidad al interior de las escuelas estigmatizadas como de mala calidad, para romper con ello, es urgente generar posibilidad desde los propios sujetos y sus comunidades. Proponen recuperar la capacidad de construir sueños y utopías, para 
desarrollar una cultura de la posibilidad desde y con los propios sujetos situados.

- Existe una fuerte demanda de participación desde las voces del profesorado, quienes quieren formar parte de un diálogo igualitario, argumentado desde su sabiduría experiencial en la diversidad de contextos laborales de la región, y desde donde contribuir con sus propias propuestas de mejora educativa. En base a lo cual se puede concluir que se busca fortalecer sus propias capacidades para coordinarse por medio del diálogo libre de coersiones y siempre susceptible de crítica, como base de la comunicación entre los sujetos situados.

- También es posible observar que el profesorado vincula permanentemente su saber especializado que porta por su formación profesional con aquel saber dado por la experiencia de largos años en desempeño laboral en diversidad de contextos, desde lo cual ha desarrollado propuestas de mejoras originales dado que no porta consigo las categorías tradicionales del investigador clásico que desconoce el saber experiencial como conocimiento científico. En palabras de Zemelman (2001), esta vinculación que realiza el profesorado viene a romper con el pensamiento teórico que reproduce modelos que explican la realidad y la fuerzan a comportarse como tal, en vez de problematizar la realidad sin tales modelos, cuestión que permitiría desarrollar un pensamiento epistémico que tanta falta nos hace en América Latina. De allí, cabe afirmar que coindicen con la necesidad de romper las jerarquías epistémicas entre el saber científico y el saber experiencial de los sujetos situados, resultado de cuya síntesis, sea posible movilizar procesos de transformación.

\section{Referencias}

Agencia de calidad de la educación. (2016) SIMCE. Recuperado de http://www.agenciaeducacion.cl/evaluaciones/simce/

Bartolomé, M. A. (2006). Los laberintos de la identidad: Procesos identitarios en las poblaciones indígenas. Avá, 9, 28-48.

Círculo de Economía. (2012). Un concepto de calidad. Círculo de economía de la provincia de Alicante. Recuperado de http://www.circuloeconomiaalicante.com/blog/un-concepto-de-calidadla-calidad-segunda-entrega-3-01-2012/

Durán, T., Catriquir, D. y Berho, M. (2011). Diversidad cultural e interculturalidad en una Universidad del centro Sur de Chile. Validando una categoría analítica. Cuadernos Interculturales, 9(17), 135-159, 2011.

Ferrada, D., Villena, A., Catriquir, D., Turra, O., Pozo, G., Schilling, C. y Del Pino, M. (2014). Investigación dialógica-kishu kimkelay ta che En educación. Revista REXE, 13(26), 33-50.

Ferrada, D., Villena, A. y Turra, O. (2015). Transformar la formación. Las voces del profesorado. Santiago: RIL Editores.

Gayá Wicks, P. y Reason, P. (2009). Initiating action research: Challenges and paradoxes of opening communicative space. Action Research, 7(3), 243-262. doi: $10.1177 / 1476750309336715$ 
Guba, E. y Lincoln, I. (2012). Controversias paradigmáticas, contradicciones y confluencias emergentes. En F. Denzin e I. Lincoln (Coords.), Paradigmas y perspectivas en disputa. Manual de investigación cualitativa (pp. 38-78). Barcelona: Gedisa.

Habermas, J. (1999). La teoría de la acción comunicativa. Madrid: Editorial Taurus.

Heron, J. y Reason, P. (1997). A participatory inquiry paradigm. Qualitative Inquiry, 3(3), 274-294. doi: $10.1177 / 107780049700300302$

JUNAEB. (2016). Junta nacional de auxilio escolar y becas. Recuperado de https://www.junaeb.cl/?s

Mesa sobre ley 20.501(2016). Informe ejecutivo de resultados y propuestas. Condiciones para la docencia $y$ agobio laboral. Recuperado de http://www.mineduc.cl/wpcontent/uploads/sites/19/2016/03/Info-Mesa-de-Condiciones-Agobio-23-FEB-2016.pdf

Navarrete, Z. (2008). Proceso de construcción identitaria del pedagogo universitario en México. Cadernos de Pesquisa, 38(134), 503-533. doi:10.1590/So100-15742008000200012

Pirrone, G. (2006). Los procesos identitarios en espacios de participación no tradicionales. Question, 11(1), 183-199.

Reyes, L., Cornejo, R., Arévalo, A. y Sánchez, R. (2010). Ser docente y subjetividad histórica en el Chile actual: discursos, prácticas y resistencias. Polis, 9(27), 269-292. doi:10.4067/S071865682010000300012

Salinas, I. y Insunza, J. (2013). ¿Por qué alto Al SIMCE? Razones humanas, políticas y pedagógicas. Revista Perspectiva, 4, 1-16.

Toledo, M. A. (2012). Sobre la construcción identitaria. Atenea, 506, 43-56. doi:10.4067/S071804622012000200004

Zemelman, H. (2001). Pensar teórico y pensar epistémico. Los retos de las ciencias sociales latinoamericanas. Ciudad de Mexico: IPECAL.

Zemelman, H. (2005). Voluntad de conocer: El sujeto y su pensamiento en el paradigma crítico. Ciudad de Mexico: Anthropos.

\section{Breve CV de la autora}

\section{Donatila Ferrada}

Chilena, Académica de la Universidad Católica del Maule. Dra. En Filosofía, mención Currículum e Interculturalidad, Magister en Educación, mención Currículum Educacional. Ha dirigido numerosos proyectos de investigación en las líneas de Formación docente, Innovación Curricular y Política educativa. Coordinadora General del Grupo para la Promoción y Acción de la Igualdad Educativa Enlazador de Mundos. Autora de los libros: Currículum crítico comunicativo, Barcelona, El Roure, 2001; Construyendo escuela compartiendo esperanzas, Santiago, RIL Editores, 2012; transformar la formación. Las voces del profesorado, Santiago, RIL Editores, 2015; y de numerosos artículos en revistas de corriente principal y capítulos de libros. Imparte docencia en pre y postgrado. ORCID ID: 0000-0003-0942-4320. Email: dferrada@ucm.cl 